\title{
Musgos del Parque Nacional los Mármoles, Hidalgo, MÉxICO
}

\author{
Claudio Delgadillo-Moya ${ }^{1,3}$, María de los Ángeles Cárdenas-Soriano", \\ Víctor Manuel Gálvez-Aguilar ${ }^{2}$ y Arturo Sánchez-GonzálezZ² \\ ${ }^{1}$ Departamento de Botánica, Instituto de Biología, Universidad Nacional Autónoma de México \\ ${ }^{2}$ Centro de Investigaciones Biológicas, Universidad Autónoma del Estado de Hidalgo \\ ${ }^{3}$ Autor para la correspondencia: moya@servidor.unam.mx
}

\begin{abstract}
Resumen: El Parque Nacional Los Mármoles, en el estado de Hidalgo, México, es una zona de interés florístico y fitogeográfico. Para proponer medidas para su conservación se debe tener un conocimiento adecuado de la biota, en particular de grupos como el de los musgos que han sido poco estudiados. Para esta investigación se recolectaron 573 muestras en los principales tipos de vegetación, que representan 129 especies y variedades de musgos. Mediante estimadores de riqueza no paramétricos (Jackknife y Bootstrap) se determinó que el inventario contiene entre el 78 y el $87 \%$ de los taxones infragenéricos esperados. La flora de musgos incluye especies como Aloina rigida y Claopodium pellucinerve que amplían su distribución conocida hacia el sur, y Campylopus anderssonii que la extiende hacia el norte. Los taxones muestran varios patrones de distribución, enunciados en orden de importancia: amplia distribución, mesoamericano, del Caribe, boreal, endémico, austral y chihuahuense. De los cuatro municipios representados en el parque, Zimapán, el de mayor extensión, también muestra la mayor riqueza de musgos (90 especies y variedades), mientras que en el de Pacula, el de menor superficie, sólo se conocen 39 taxones.
\end{abstract}

Palabras clave: área natural protegida, Hidalgo, Los Mármoles, musgos.

\begin{abstract}
Los Mármoles National Park, in the Mexican state of Hidalgo, is an area of floristic and phytogeographical interest. An adequate knowledge of its biota is a requisite before adopting conservation measures, especially for locally unknown plant groups such as the mosses. For this research, 573 specimens representing 129 species and varieties of mosses, were obtained from the main types of vegetation. Non-parametric richness estimators (Jackknife and Bootstrap), indicate that the local moss inventory contains between 78 and $87 \%$ of the expected infrageneric taxa. The moss flora includes such species as Aloina rigida and Claopodium pellucinerve that extend southwards their known distribution, and Campylopus anderssonii that extends it northwards. The identified patterns of distribution are arranged by number of species, as follows: Wide distribution, Mesoamerican, Caribbean, Boreal, Endemic, Southern, and Chihuahuan. Zimapán, the largest of the four municipalities represented in the park, is the most diverse with 90 moss species and varieties; in contrast, Pacula, with the smallest surface area, has the smallest moss flora, with 39 taxa.
\end{abstract}

Key words: Hidalgo, Los Mármoles, mosses, natural protected area.

$\mathbf{S}^{\mathrm{e}}$ gún la Comisión Estatal de Ecología (COEDE) del estado de Hidalgo, hasta el tres de abril de 2008, existían 38 áreas naturales protegidas de carácter federal, estatal y municipal en esa entidad (Comunicación de las Huastecas, 2008). Entre ellas, la segunda en importancia por su extensión territorial, es el Parque Nacional Los Mármoles (PNM) el cual, a pesar de su categoría y designación, hasta ahora no cuenta con disposiciones específicas para su conservación, manejo o aprovechamiento. Esta situación preocupa a los que se oponen a la derogación del decreto que lo creó en 1936, pues la aprobación de un cambio podría dar lugar a la

transformación de zonas forestales a agrícolas, la expansión de los asentamientos humanos, la erosión de suelo y el aumento de la explotación de mármol, con el consiguiente deterioro ambiental y la pérdida de la biodiversidad. Aunque el número de taxones en la biota del PNM todavía es motivo de estudio por grupos de investigación de la Universidad Autónoma del Estado de Hidalgo, se presupone que es alto, pues el área está situada en la zona de contacto de la Sierra Madre Oriental y el Eje Neovolcánico, lo que la hace potencialmente muy diversa.

La preservación de la biota de cualquier área presupo- 
ne el conocimiento de los organismos que la habitan. En el caso de los musgos del PNM la información florística es insuficiente por provenir de algunas colecciones incidentales (CONANP, 2007). Por ello, a través de información de campo, se desea establecer con mayor precisión el número de especies de ese grupo de plantas en la flora local. Se ha establecido que los musgos reducen la erosión, intervienen en el intercambio de iones, participan (con cianobacterias) en la fijación de nitrógeno, son refugio para numerosos invertebrados, zona de germinación para semillas de muchas especies de plantas vasculares (Ando y Matsuo, 1984; Glime y Saxena, 1991) y son considerados indicadores de condiciones ambientales (Frego, 2007; Fritz et al., 2009). El primer paso para saber cómo participan los musgos en la economía del PNM, es obtener una evaluación preliminar sobre su riqueza y distribución, para luego considerar los resultados en las decisiones sobre su conservación y manejo. La importancia de dicha zona también se puede apreciar por la peculiaridad de las relaciones geográficas de sus elementos; por esta razón, en este estudio se revisa la distribución geográfica general de las especies.

\section{Material y métodos}

Área de estudio. El Parque Nacional Los Mármoles se localiza entre los $20^{\circ} 45^{\prime} 39^{\prime \prime}-20^{\circ} 58^{\prime} 22^{\prime \prime} \mathrm{N}$ y los $99^{\circ} 08^{\prime}$ 57" - 99 18' 39" W (Figura 1), a $126 \mathrm{~km}$ al noroeste de la ciudad de Pachuca. De las 138,793 hectáreas de áreas naturales protegidas del estado de Hidalgo, 23,150 corresponden al parque, distribuidas en los municipios de Jacala de Ledesma, Nicolás Flores, Pacula y Zimapán. Como parte de la Sierra Gorda, el PNM cuenta con desniveles topográficos importantes como la Barranca de San Vicente, con una profundidad de $600 \mathrm{~m}$, y el Cerro del Cangandó que alcanza una altitud de 3,000 m (SARH, 1994). El parque está incluido dentro de la Cuenca del Bajo Río Pánuco, los arroyos que drenan hacia el occidente forman el río Barranca Seca, que hacia el norte se une al río Moctezuma. En el extremo oriente se forman los ríos San Nicolás e Itatlaxco que drenan al oriente hacia el río Amajac, el que a su vez hacia el norte, se une al río Moctezuma (SARH, 1994). En el PNM se presentan dos tipos de clima: templado sub-húmedo (el dominante) y semicálido sub-húmedo, ambos con lluvias

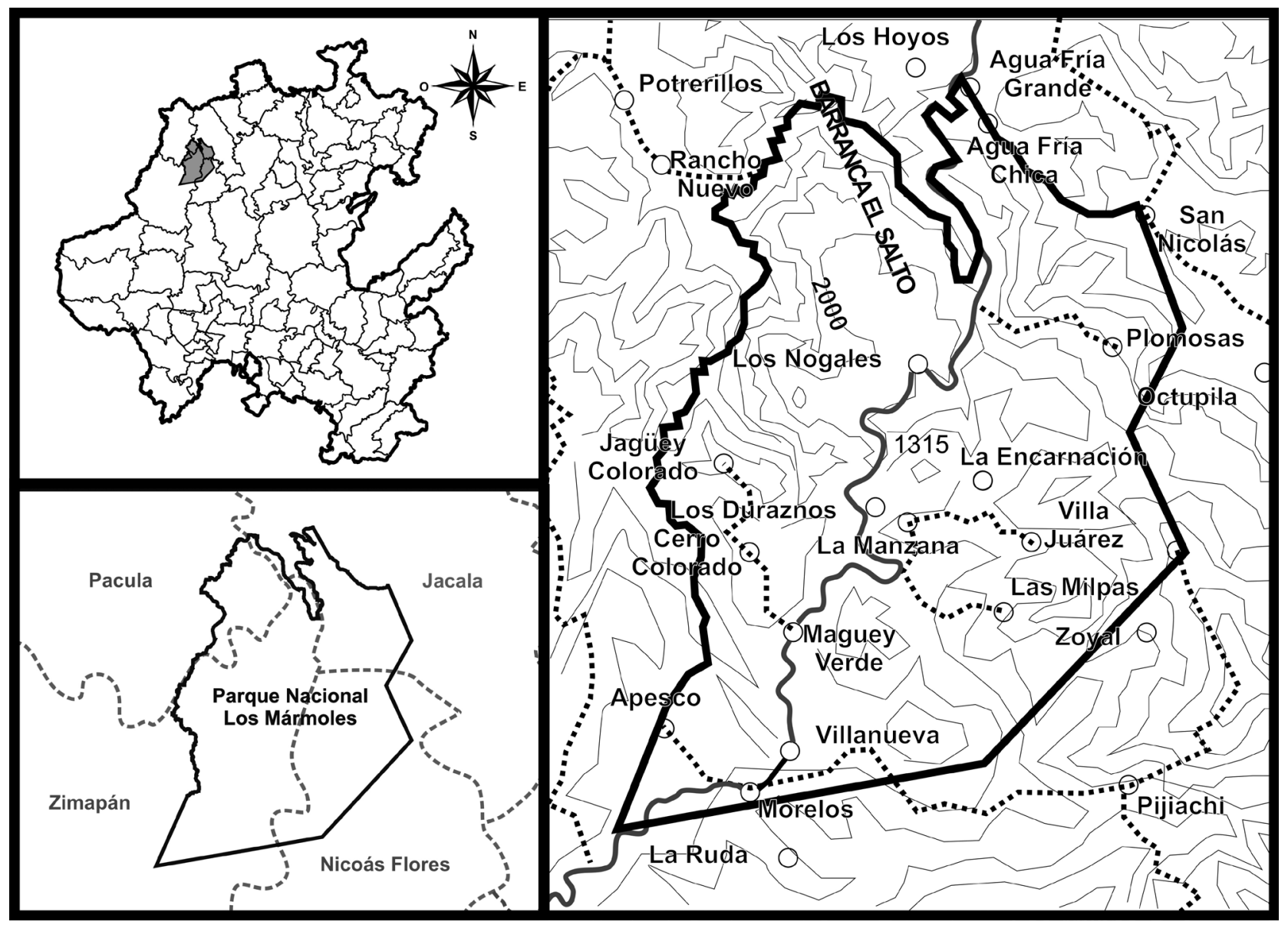

Figura 1. Área de estudio (tomado de Ramírez et al., 2009) 
Cuadro 1. Características principales de los municipios en donde se encuentra el Parque Nacional Los Mármoles, estado de Hidalgo. Extensión territorial y clima con base en CONANP (2007); los tipos de vegetación por municipio, con base en Sánchez-González et al. (2010).

\begin{tabular}{lll}
\hline Municipio & $\begin{array}{l}\text { Extensión dentro } \\
\text { del PNM y clima } \\
\text { dominante }\end{array}$ & Tipos de vegetación \\
\hline $\begin{array}{l}\text { Jacala } \\
\text { de Ledesma }\end{array}$ & $\begin{array}{l}7,986.75 \text { ha }(34.5 \%) \\
\text { Semicálido sub-húmedo }\end{array}$ & $\begin{array}{l}\text { Bosque de } \\
\text { Juniperus-Quercus }\end{array}$ \\
Nicolás Flores & $\begin{array}{l}5,787.5 \text { ha }(25.0 \%) \\
\text { Templado sub-húmedo }\end{array}$ & $\begin{array}{l}\text { Bosque de Juniperus, } \\
\text { bosque de Quercus, } \\
\text { bosque de Pinus, } \\
\text { vegetación secundaria }\end{array}$ \\
Pacula & $\begin{array}{l}\text { 1,041.75 ha (4.5\%) } \\
\text { Semicálido sub-húmedo }\end{array}$ & $\begin{array}{l}\text { Bosque de Quercus, } \\
\text { vegetación secundaria }\end{array}$ \\
& $\begin{array}{l}\text { 8,334.0 ha (36.0\%) } \\
\text { Zimapán }\end{array}$ & $\begin{array}{l}\text { Bosque de Quercus, } \\
\text { bosque de Pinus-Quercus, } \\
\text { bosque de Pinus, } \\
\text { vegetación secundaria }\end{array}$ \\
\hline
\end{tabular}

de verano (Cuadro 1). La temperatura anual promedio se encuentra entre los $14{ }^{\circ} \mathrm{C}$ en la región seca y $19{ }^{\circ} \mathrm{C}$ en la cálida al norte y el régimen de lluvia tiene un gradiente de 450 , hasta cerca de los $1,500 \mathrm{~mm}$ anuales, respectivamente (CONANP, 2007). Este gradiente higrotérmico se establece debido a que el PNM se localiza hacia el lado de sotavento de la Sierra Madre Oriental. La vegetación dominante incluye los siguientes tipos, de acuerdo a la nomenclatura de Rzedowski (1978): bosque de Quercus (en el que dominan Quercus affinis, $Q$. crassifolia, $Q$. laeta y $Q$. mexicana), bosque de Pinus-Quercus, bosque de Pinus (representado por dos asociaciones importantes: el bosque de Pinus cembroides asociado con Juniperus deppeana y el bosque de Pinus greggii, $P$. patula, $P$. pseudostrobus y $P$. teocote), bosque de Juniperus (dominado por Juniperus deppeana y J. flaccida), matorral xerófilo (Acacia berlandieri, Caesalpinia mexicana, Dalea bicolor, Karwinskia humboldtiana, Mimosa aculeaticarpa y Senna racemosa), vegetación secundaria y pastizal inducido (Sánchez-González et al., 2010).

Trabajo en campo. Entre el 30 de septiembre de 2005 y el 17 de diciembre de 2006 se recolectaron 573 ejemplares de musgos, en los principales tipos de vegetación del área de estudio. Los ejemplares fueron recolectados en 46 puntos de muestreo, localizados dentro de nueve localidades principales (Barranca San Vicente, Camposanto, Cerro Cangandó, Jacala, La Encarnación, Maguey Verde, Nicolás Flores, Pacula y Puerto de Piedra), con énfasis en el municipio de Zimapán, que es el de mayor superficie de los cuatro en los que está comprendido el parque.

Trabajo de laboratorio. La determinación de los especímenes se realizó mediante el manual de la Flora de los musgos de México (Sharp et al., 1994) y revisiones taxonómicas recientes. La nomenclatura utilizada y la distribución de los taxones de musgos del PNM se basó en la versión digital actualizada de LATMOSS <www.ibiologia.unam.mx/ briologia/www/index/>, una base de datos desarrollada por Delgadillo et al. (1995) que contiene información sobre la distribución mundial de las especies de musgos del neotrópico. Un juego completo de los ejemplares determinados fue depositado en la Colección de Briofitas del Herbario Nacional (MEXU), con duplicados en la colección de briofitas del herbario HGOM de la Universidad Autónoma del Estado de Hidalgo.

Para determinar el grado de completitud del inventario en el PNM, se comparó la riqueza de taxones infra-genéricos (especies y variedades) de musgos, observada versus la esperada, con base en métodos no paramétricos (Colwell y Coddington, 1994), se utilizaron los estimadores basados en incidencia Bootstrap y Jackknife (Chazdon et al., 1998). Se estimó también la tasa de reemplazo de especies entre municipios (diversidad beta). Los valores se obtuvieron con el programa de cálculo EstimateS versión 7.5 (Colwell, 2006). La diversidad beta se estimó como el inverso del índice de semejanza de Sørensen.

\section{Resultados}

La flora de musgos del Parque Nacional Los Mármoles comprende alrededor de 129 especies y variedades (Cuadro 2), es decir, casi el $46 \%$ de la flora de musgos reconocida por Sharp et al. (1994) para el estado de Hidalgo (281 especies). Su distribución mundial permite reconocer los siguientes patrones, en orden de mayor a menor número de especies: amplia distribución, mesoamericano, del Caribe, boreal, endémico, austral y chihuahuense (Cuadro 2, Figura 3 ). Los musgos de amplia distribución son conocidos por registros en varios continentes. En el parque éstos constituyen el grupo más numeroso con 56 taxones, o $43 \%$ del total de la flora de musgos local, entre los que hay cuatro cosmopolitas (Bryum argenteum, Funaria hygrometrica var. hygrometrica, Polytrichum juniperinum y Weissia controversa), especies bicontinentales (p. ej., Pilotrichella flexilis y Platygyriella densa), pantropicales (p. ej., Bryum billarderi y Palamocladium leskeoides) y otras cuya distribución es más amplia, en dos o más continentes.

Las especies mesoamericanas son exclusivamente americanas; su área de distribución se extiende principalmente de México al norte o centro de Sudamérica, pero sin incluir a las Antillas. Son ejemplo de este patrón de distribución Brachythecium occidentale, Bryum procerum y Rauiella lagoensis. En comparación con éstas, las especies del Caribe ocupan la misma área, pero también están presentes en las islas del Caribe. Campylopus tallulensis, Ptychomitrium lepidomitrium y Sematophyllum swartzii pertenecen a este último grupo. Por su parte, las especies denominadas 
Cuadro 2. Musgos del Parque Nacional Los Mármoles, sus patrones de distribución y municipios en los que se distribuyen dentro del área de estudio. J = Jacala de Ledesma, $\mathrm{N}=$ Nicolás Flores, $\mathrm{P}=$ Pacula, $\mathrm{Z}=$ Zimapán.

\begin{tabular}{|c|c|c|c|}
\hline Taxones/Distribución & Municipio & Taxones/Distribución & Municipio \\
\hline Amplia Distribución & & Pohlia elongata Hedw. & Z \\
\hline Aloina rigida (Hedw.) Limpr. & Z & Polytrichum juniperinum Hedw. & $\mathrm{JZ}$ \\
\hline Anacolia laevisphaera (Taylor) Flowers & Z & Prionodon densus (Hedw.) Müll. Hal. & NPZ \\
\hline Barbula convoluta Hedw. & $\mathrm{Z}$ & Pseudocrossidium replicatum (Taylor) R.H. Zander & NJPZ \\
\hline Brachymenium exile (Dozy \& Molk.) Bosch \& Lac. & Z & Pylaisia falcata Schimp. & NZ \\
\hline Brachymenium systylium (Müll. Hal.) A. Jaeger & NP & Pylaisia polyantha (Hedw.) Schimp. & $\mathrm{N}$ \\
\hline Brachythecium ruderale (Brid.) W.R. Buck & $\mathrm{PPZ}$ & Racopilum tomentosum (Hedw.) Brid. & $J Z$ \\
\hline Braunia secunda (Hook.) Bruch \& Schimp. & Z & Rhabdoweisia fugax (Hedw.) Bruch \& Schimp. & Z \\
\hline Bryoerythrophyllum campylocarpum (Müll. Hal.) H.A. Crum & NZ & Sematophyllum adnatum (Mx.) E. Britton & NJZ \\
\hline Bryum argenteum Hedw. & $\mathrm{PZ}$ & Sematophyllum subpinnatum (Brid.) E. Britton & NJ \\
\hline Bryum billarderi Schwägr. & JNPZ & Sematophyllum subsimplex (Hedw.) Mitt. & $\mathrm{N}$ \\
\hline Bryum pallescens Schleich. ex Schwägr. & Z & Weissia controversa Hedw. & $J$ \\
\hline Campylopus flexuosus (Hedw.) Brid. & NZ & Zygodon obtusifolius Hook. & Z \\
\hline Campylopus nivalis (Brid.) Brid. & NZ & Boreal & \\
\hline Campylopus pilifer Brid. & NZ & & \\
\hline Campylopus savannarum (Müll. Hal.) Mitt. & J & Anomodon rostratus (Hedw.) Schimp. & $\mathrm{P}$ \\
\hline Ceratodon purpureus subsp. stenocarpus & Z & Anomodon thraustus Müll. Hal. & $\mathrm{P}$ \\
\hline (Bruch \& Schimp.) Dixon & & Atrichum angustatum (Brid.) Bruch \& Schimp. & $J Z$ \\
\hline Cyrto-hypnum minutulum (Hedw.) W.R. Buck \& H.A. Crum & JN & Bryum erythroloma (Kindb.) Syed & $\mathrm{N}$ \\
\hline Didymodon australasiae (Hook. \& Grev.) R.H. & Z & Campyliadelphus chrysophyllus (Brid.) Kanda & $J P$ \\
\hline Zander var. australasiae & & Campylophyllum sommerfeltii (Myr.) Hedenäs & NJZ \\
\hline Didymodon ferrugineus (Schimp. ex Besch.) M.O. Hill & Z & Claopodium pellucinerve (Mitt.) Best & $\mathrm{P}$ \\
\hline Didymodon rigidulus var. gracilis (Schleich. ex Hook.Grev.) & JNPZ & Haplocladium angustifolium (Hampe \& Müll. Hal.) Broth. & NJPZ \\
\hline R.H. Zander & & Haplocladium microphyllum (Hedw.) Broth. & J \\
\hline Didymodon rigidulus var. icmadophilus (Schimp. & $\mathrm{N}$ & Molendoa sendtneriana (Bruch \& Schimp.) Limpr. & NPZ \\
\hline ex Müll. Hal.) R.H. Zander & 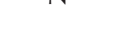 & Orthotrichum Iyellii Hook. \& Taylor & $\mathrm{N}$ \\
\hline Didymodon rigidulus Hedw. var. rigidulus & PZ & Pylaisiadelpha tenuirostris (Bruch \& Schimp.) W.R. Buck & Z \\
\hline Entodon macropodus (Hedw.) Müll. Hal. & Z & Rhynchostegium serrulatum (Hedw.) A. Jaeger & NZ \\
\hline Fissidens asplenioides Hedw. & Z & Syntrichia fragilis (Taylor) Ochyra & Z \\
\hline Forsstroemia producta (Hornsch.) Par. & NP & Thuidium delicatulum (Hedw.) Schimp. var. delicatulum & $\mathrm{N}$ \\
\hline Funaria hygrometrica var. calvescens (Schwägr.) Mont. & Z & Thuidium delicatulum var. radicans (Kindb.) & NZ \\
\hline Funaria hygrometrica Hedw. var. hygrometrica & $\mathrm{Z}$ & H.A. Crum, Steere \& Anders. & \\
\hline Gymnostomum aeruginosum Sm. & Z & Timmiella anomala (Bruch \& Schimp.) Limpr. & NZ \\
\hline Groutiella tomentosa (Hornsch.) Wijk \& Marg. & J & Tortella tortuosa (Hedw.) Limpr. & $\mathrm{JN}$ \\
\hline Hedwigia ciliata (Hedw.) P. Beauv. & $\mathrm{N}$ & Trichostomum crispulum Bruch & NZ \\
\hline Herpetineuron toccoae (Sull. \& Lesq.) Cardot & $\mathrm{PZ}$ & Trichostomum tenuirostre (Hook. \& Taylor) Lindb. & Z \\
\hline Hypnum cupressiforme Hedw. var. cupressiforme & Z & Mesoamericano & \\
\hline Leptodontium flexifolium (Dicks. ex With.) Hampe & Z & & \\
\hline Leptodontium viticulosoides (P. Beauv.) Wijk & NZ & Atrichum oerstedianum (Müll. Hal.) Mitt. & NZ \\
\hline \& Margad. var. viticulosoides & & Brachythecium cirriphylloides McFarland & Z \\
\hline Macrocoma orthotrichoides (Raddi) Wijk \& Margad. & NPZ & Brachythecium occidentale (Hampe) A. Jaeger & JNZ \\
\hline Macrocoma tenuis subsp. sullivantii (Müll. Hal.) Vitt & NJPZ & Braunia squarrulosa (Hampe) Müll. Hal. & $J \mathrm{Z}$ \\
\hline Mittenothamnium reptans (Hedw.) Cardot & NJPZ & Bryum procerum Schimp. ex Besch. & NZ \\
\hline Orthotrichum anomalum Hedw. & Z & Bryum richardsii Sharp & NPZ \\
\hline Palamocladium leskeoides (Hook.) E. Britton & NPZ & Campylopus anderssonii (Müll. Hal.) A. Jaeger & JNZ \\
\hline Papillaria nigrescens (Hedw.) A. Jaeger & J & Entodon jamesonii (Taylor) Mitt. & NZ \\
\hline Pilotrichella flexilis (Hedw.) Aongstr. & JNPZ & Flowersia campylopus (Schimp.) D.G. Griffin \& W.R. Buck & Z \\
\hline Platygyriella densa (Hook.) W.R. Buck & $\mathrm{N}$ & Leucodon curvirostris Hampe & JNPZ \\
\hline Pleurochaete squarrosa (Brid.) Lindb. & JNPZ & Leskea angustata Taylor & Z \\
\hline
\end{tabular}




\begin{tabular}{|c|c|}
\hline Taxones/Distribución & Municipio \\
\hline Lindbergia mexicana (Besch.) Cardot & $\mathrm{PZ}$ \\
\hline Meteorium teres Mitt. & NZ \\
\hline Neckera chlorocaulis Müll. Hal. & NJPZ \\
\hline Neckera ehrenbergii Müll. Hal. & Z \\
\hline Oreoweisia delgadilloi H. Rob. \& F.D. Bowers & Z \\
\hline Orthotrichum pycnophyllum Schimp. ex Müll. Hal. & NPZ \\
\hline Pterobryopsis mexicana (Renauld \& Cardot) M. Fleisch. & $\mathrm{P}$ \\
\hline Ptychomitrium serratum (Müll. Hal.) Bruch \& Schimp. & $J P Z$ \\
\hline Rauiella lagoensis (Hampe) W.R. Buck & Z \\
\hline Rhexophyllum subnigrum (Mitt.) Hilp. & Z \\
\hline Rhynchostegium subrusciforme (Müll. Hal.) A. Jaeger & $\mathrm{P}$ \\
\hline Rozea andrieuxii (Müll. Hal.) Besch. var. andrieuxii & Z \\
\hline Sphaerotheciella pachycarpa (Schimp. ex Besch.) Manuel & $\mathrm{N}$ \\
\hline Syntrichia obtusissima (Müll. Hal.) R.H. Zander & $\mathrm{PZ}$ \\
\hline Thuidium delicatulum var. peruvianum (Mitt.) H.A. Crum & NJPZ \\
\hline Zygodon ehrenbergii Müll. Hal. & Z \\
\hline \multicolumn{2}{|l|}{ Del Caribe } \\
\hline Barbula orizabensis Müll. Hal. & Z \\
\hline Brachymenium mexicanum Mont. & $\mathrm{P}$ \\
\hline Breutelia brittoniae Renauld \& Cardot & Z \\
\hline Campylopus tallulensis Sull. \& Lesq. & $\mathrm{N}$ \\
\hline Cryphaea patens Hornsch. & NJP \\
\hline Entodon beyrichii (Schwägr.) Müll. Hal. & NPZ \\
\hline Entodon hampeanus Müll. Hal. & $\mathrm{N}$ \\
\hline Entosthodon obtusifolius Hook f. in Hook. & $\mathrm{N}$ \\
\hline Erythrodontium longisetum (Hook.) Par. & $\mathrm{JP}$ \\
\hline Hypnum amabile (Mitt.) Hampe & $\mathrm{N}$ \\
\hline $\begin{array}{l}\text { Leptodontium viticulosoides var. sulphureum } \\
\text { (Müll. Hal.) R.H. Zander }\end{array}$ & $\mathrm{JZ}$ \\
\hline Leucobryum albidum (P. Beauv.) Lindb. & NP \\
\hline Leucodon julaceus (Hedw.) Sull. & $J P$ \\
\hline Macromitrium guatemalense Müll. Hal. & $\mathrm{P}$ \\
\hline Meteorium illecebrum Sull. & NJPZ \\
\hline Papillaria deppei (Hornsch. ex Müll. Hal.) A. Jaeger & $J P$ \\
\hline Pogonatum campylocarpum (Müll. Hal.) Mitt. & JNPZ \\
\hline Ptychomitrium lepidomitrium (Müll. Hal.) Schimp. & NJZ \\
\hline Rhynchostegium scariosum (Taylor) A. Jaeger & Z \\
\hline Sematophyllum cuspidiferum Mitt. & $\mathrm{PZ}$ \\
\hline Sematophyllum swartzii (Schwägr.) Welch \& H.A. Crum & NZ \\
\hline Zygodon campylophyllus Müll. Hal. & $\mathrm{PZ}$ \\
\hline
\end{tabular}

Endémico

Entodon abbreviatus (Besch.) A. Jaeger

Homomallium sharpii Ando \& Higuchi

Chihuahuense

Homomallium mexicanum Cardot

Austral

Erpodium beccarii Müll. Hal. ex Vent.
Cuadro 3. Grado de completitud del inventario de taxones de musgos del Parque Nacional Los Mármoles, evaluado con dos estimadores

\begin{tabular}{lccc}
\hline & & \multicolumn{2}{c}{ Riqueza esperada } \\
\cline { 3 - 4 } & $\begin{array}{c}\text { Riqueza } \\
\text { observada }\end{array}$ & Jackknife & Bootstrap \\
\hline Riqueza de especies & 129 & 166 & 148 \\
Porcentaje de completitud & - & 78 & 87 \\
\hline
\end{tabular}

boreales son aquellas cuya distribución principal ocurre en masas continentales del hemisferio norte. Campyliadelphus chrysophyllus, Claopodium pellucinerve, Haplocladium angustifolium y Molendoa sendtneriana son ejemplos de este patrón.

Algunas especies muestran áreas de distribución singular; tal es el caso de Erpodium beccarii el cual se conoce de México hasta Argentina, del NE, E y S África y estaciones en el Océano Índico Occidental (Delgadillo et al., 1995; Pursell y Allen, 2002). Por ello se le considera como un taxón austral y es el único de este tipo en el PNM. Por su parte, Homomallium mexicanum se ha designado tentativamente como Chihuahuense pues su área de distribución principal parece centrarse en el suroeste de los Estados Unidos y en los estados de Chihuahua, Coahuila y Durango. Sin embargo, Sharp et al. (1994) lo ubican en Guanajuato, Hidalgo y Oaxaca. El contingente endémico también está pobremente representado pues sólo se han detectado dos especies, Entodon abbreviatus y Homomallium sharpii, con áreas de distribución restringida a México.

Aunque la flora de musgos del parque podría considerarse como proporcionalmente rica, los estimadores de riqueza de especies utilizados (Cuadro 3, Figura 2), indican que to-

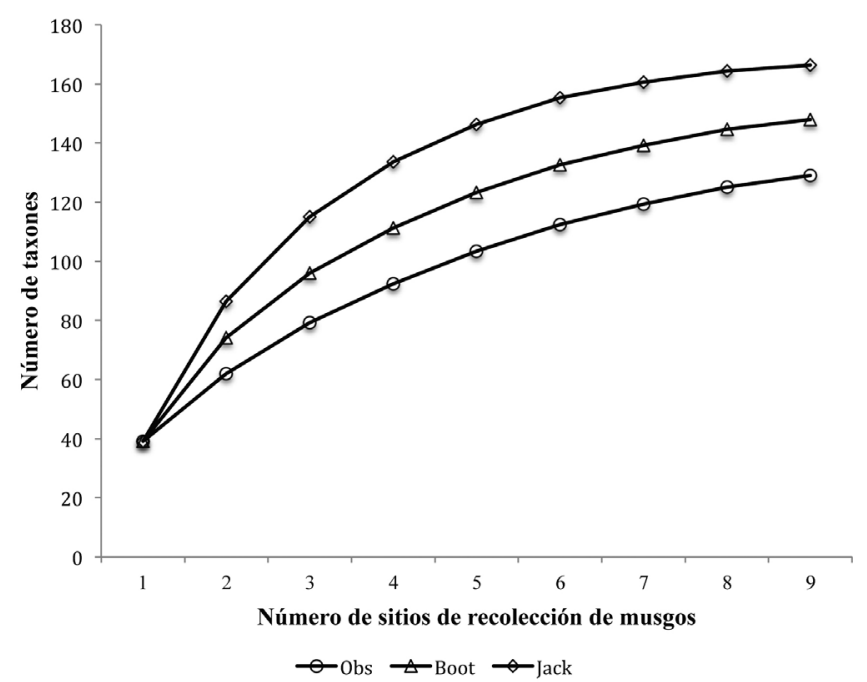

Figura 2. Riqueza de taxones de musgos observada (Obs) y esperada, con base en los estimadores no para-métricos Jackknife (Jack) y Bootstrap (Boot). 
Cuadro 4. Número de especies compartidas (parte superior derecha), riqueza de especies de musgos (en negrita, en diagonal) e índice de diversidad beta (parte inferior izquierda) de municipios que comprende el Parque Nacional Los Mármoles

\begin{tabular}{lcccc}
\hline Municipio & Zimapán & Nicolás Flores & Pacula & Jacala \\
\hline Zimapán & $\mathbf{9 0}$ & 38 & 30 & 25 \\
Nicolás Flores & 0.50 & $\mathbf{6 1}$ & 25 & 23 \\
Pacula & 0.44 & 0.46 & $\mathbf{4 7}$ & 21 \\
Jacala & 0.39 & 0.46 & 0.49 & $\mathbf{3 9}$ \\
\hline
\end{tabular}

davía no está completamente caracterizada. La completitud del inventario fluctuó entre 78\% (con el estimador Jackknife) y $87 \%$ (con el estimador Bootstrap), es decir, el esfuerzo de muestreo fue suficiente para contar con un inventario adecuado de los musgos del PNM, pero se deben esperar adiciones a la flora en otros trabajos de campo.

Localmente, el municipio de Zimapán, con la mayor extensión territorial del PNM (36\%), muestra la mayor riqueza de taxones de musgos, mientras que el municipio de Pacula, con sólo el $4.5 \%$ de la superficie del parque (CONANP, 2007), ofrece la menor riqueza florística (Cuadro 4). La tasa de reemplazo de especies (diversidad beta) entre estos municipios, concuerda con el fenómeno de decaimiento con la distancia, según el cual los sitios (municipios) geográficamente más cercanos, son en promedio más semejantes entre sí en la composición de taxones (y en las características ambientales) que los sitios (municipios) más distantes ( $c f$. Tuomisto et al., 2003).

\section{Discusión}

El inventario preliminar de la flora de musgos del PNM es una adición importante al conocimiento de la diversidad

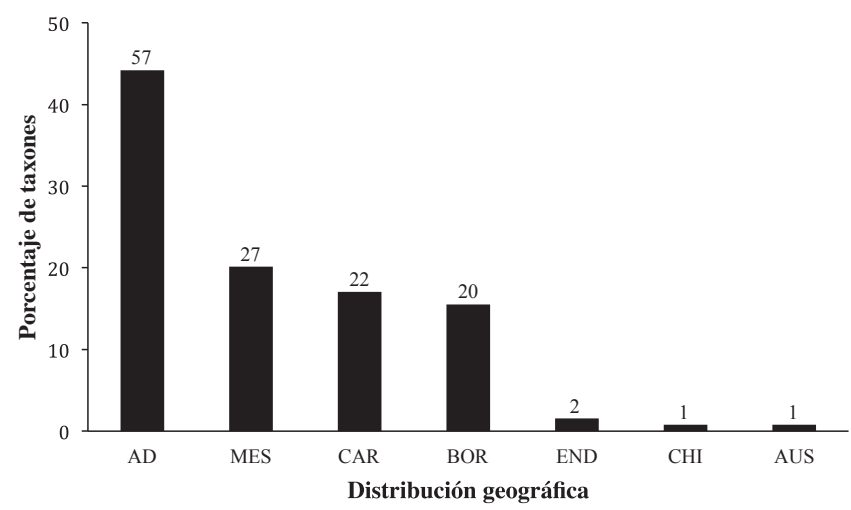

Figura 3. Distribución geográfica conocida de los musgos del Parque Nacional Los Mármoles, estado de Hidalgo, México. Sobre cada barra se indica el número de taxones, AD: Amplia distribución, MES: Mesoamericano, CAR: Caribe, BOR: Boreal, END: Endémico, CHI: Chihuahuense, AUS: Austral. del estado de Hidalgo porque son pocas las contribuciones existentes sobre los musgos de esa entidad. En su lista de musgos de México, Crum (1951) citó datos de distribución y ejemplares de Hidalgo; Delgadillo (1979), mencionó algunas especies del bosque caducifolio, y Alfaro-Omaña y Castillo-Delgadillo (1986), enlistaron las especies de musgos de la Sierra de Pachuca. En las tres áreas de estudio se necesita realizar trabajo de campo adicional, examinar el material disponible en otros herbarios y actualizar la nomenclatura de los ejemplares antiguos, para producir un catálogo confiable de las especies de musgos del estado.

La flora de musgos del PNM sólo era conocida por registros de colectas incidentales de botánicos en tránsito. En esta contribución se enlistan las especies derivadas de trabajos de campo, pero seguramente no se incluyen muchas especies raras o las que por su similitud morfológica superficial podrían haberse confundido durante la recolección. Un esfuerzo de recolecta mayor durante y al cierre de la temporada de lluvias, podría permitir la obtención de ejemplares de musgos pequeños o efímeros que actualmente no son parte de la colección. Los muestreos adicionales también podrían mejorar el conocimiento de la distribución local de especies o de grupos mal conocidos como el género Fissidens que está precariamente representado en el muestreo actual. Como la detección de especies para completar los inventarios depende directamente de su abundancia, del método de muestreo ( $c f$. Nakamura y Soberón, 2008) y de la experiencia del investigador, se debe buscar la aplicación periódica de estimadores de riqueza como una referencia estadística de los avances en el conocimiento de la riqueza florística del parque. También deben considerarse otras variables como la historia geológica y la situación geográfica para hacer un diagnóstico de las especies esperadas.

La situación geográfica de PNM en la zona de contacto entre áreas geográficas y tipos de vegetación con historias disímbolas, podría haber propiciado la presencia de especies de musgos de interés particular. Es prematuro saber el significado de los hallazgos de Aloina rigida y Claopodium pellucinerve dentro del parque. Nuestros registros extienden su distribución conocida hacia el sur en México, mientras que para Campylopus anderssonii la extienden hacia el norte. Por otra parte, aunque la flora del parque es similar a la de los bosques de Pinus y Quercus del Eje Neovolcánico, incluye representantes de especies propias de los bosques mesófilos de montaña, como Anomodon rostratus, Groutiella tomentosa y Pilotrichella flexilis. La presencia de este tipo de vegetación en municipios cercanos (p. ej., La Misión, Hidalgo), sugiere que hay especies compartidas entre el bosque mesófilo y los tipos de vegetación del parque que indican la complejidad de las relaciones de su flora. Otras especies como Forsstroemia producta y Leucodon julaceus crecen en encinares y bosques de Juniperus del noreste del país, pero también se les ha recolectado en los bosques mesófilos de montaña de Hidalgo y Querétaro. 
La diferencia en riqueza de especies y variedades entre municipios (Cuadro 4) es una situación esperada de acuerdo con la bien documentada relación especies-área según la cual, independientemente del grupo taxonómico o del tipo de ecosistema, el número de especies se incrementa conforme aumenta el tamaño del área (Whittaker et al., 2001). Sin embargo, la relación especies-área no explica la diferencia entre el municipio de Nicolás Flores, con menor extensión territorial que el de Jacala de Ledesma, pero con mayor riqueza florística. Varios factores independientes de la relación especies/área, como la productividad, la disponibilidad de energía (clima) y la heterogeneidad ambiental (Whittaker et al., 2001; Triantis et al., 2008) son críticos y podrían explicar esos patrones de riqueza de especies. Es probable que la menor riqueza florística de Jacala de Ledesma y de Pacula se explique por un paisaje relativamente homogéneo con pocos tipos de vegetación (Cuadro 1). En contraste, la mayor riqueza de taxones en Zimapán y Nicolás Flores, podría relacionarse con su topografía accidentada que da lugar a una heterogeneidad ambiental representada por varios tipos de vegetación (Cuadro 1) con diferente composición florística. En el PNM las condiciones ambientales no son homogéneas por lo que los musgos podrían tener preferencias ecológicas que se reflejan en su distribución diferencial en los municipios. El clima templado sub-húmedo predomina en los municipios de Nicolás Flores y Zimapán; en cambio, el clima semi-cálido sub-húmedo se encuentra únicamente al norte, hacia los municipios de Jacala y Pacula (SARH, 1994; CONANP, 2007).

El conocimiento de la composición y riqueza de especies de musgos es útil para propósitos de conservación en un área determinada, pero la diversidad beta es un factor más importante en el establecimiento de estrategias eficientes para la protección de áreas naturales y de especies particulares (van Dyke, 2008). La diversidad beta de una región se ha relacionado con el concepto de complementariedad, cuyo propósito es encontrar la combinación con el menor número de sitios que incluya la totalidad de especies de una región (Rodríguez et al., 2003). Como la tasa de recambio de especies es significativa entre municipios (entre $39 \%$ y $50 \%$ ), la conservación de la riqueza de musgos en el PNM depende de la protección territorial de los cuatro municipios, ya que cada uno de ellos posee una fracción de especies de musgos característica.

En lo referente al disturbio, es posible mencionar que todas las localidades en donde se recolectaron ejemplares de musgos, presentaban signos evidentes de intervención humana. Es decir, el PNM es una zona sumamente perturbada; aun sitios distantes y poco accesibles, como el Cerro Cangandó y la Barranca de San Vicente, presentan indicios de disturbio por actividades humanas. En concordancia con lo que menciona la CONANP (2007), el problema principal es el cambio de uso del suelo por la presencia de ganado (bovino y caprino, principalmente), cultivos agrícolas, minería (mármol), extracción de árboles de especies de encino y de pino, y la construcción de viviendas y caminos. Estas actividades influirán y afectarán drásticamente en el corto plazo, la presencia, abundancia y distribución de las especies de musgos (y de los demás grupos de plantas) en el parque.

Las briofitas son consideradas potencialmente como un grupo indicador de la calidad de los sitios donde habitan (Mishler, 2003; Frego, 2007; Rydin, 2008), ya que por su tamaño pequeño, carencia de hojas, raíces y tipo de reproducción sexual, tienen requerimientos micro-ambientales particulares, en especial de humedad. Por ello, tanto el conocimiento de la composición de especies de musgos, como el de su distribución dentro del PNM, constituyen una referencia útil para evaluar en el futuro cercano, el impacto de las actividades humanas sobre la calidad del hábitat.

\section{Agradecimientos}

El presente estudio contó con el apoyo económico del proyecto PROMEP "Estudio florístico, biogeográfico y sinecológico del Parque Nacional Los Mármoles, Hidalgo, México".

\section{Literatura citada}

Alfaro-Omaña A.P. y Castillo-Delgadillo X.J. 1986. Distribución por tipos de vegetación de los musgos de la Sierra de Pachuca, Hidalgo. Tesis de licenciatura, Facultad de Estudios Superiores Zaragoza, Universidad Nacional Autónoma de México. México, D.F. 55 pp.

Ando H. y Matsuo A. 1984. Applied bryology. Advances in Bryology 2:133-236.

Comunicación de las Huastecas. 2008. Cuentan [sic] Hidalgo con 38 áreas naturales protegidas: COEDE. Inforural <www. inforural.com.mx/noticias.php?\&id_rubrique=364\&id_article $=22445>$ (consultada el 15 de febrero de 2011).

Colwell R.K. 2006. EstimateS v. 8.x: statistical estimation of species richness and shared species from samples. <viceroy.eeb. uconn.edu/EstimateS $>$ (consultada el 10 de febrero de 2011)

Colwell R.K. y Coddington J.A. 1994. Estimating terrestrial biodiversity through extrapolation. Philosophical Transactions of the Royal Society (Series B) 345:101-118.

CONANP [Comisión Nacional de Áreas Naturales Protegidas]. 2007. Estudio previo justificativo para la modificación del decreto por el que se pretende re-categorizar el Parque Nacional Los Mármoles como área de protección de flora y fauna. Comisión Nacional de Áreas Naturales Protegidas-Secretaría del Medio Ambiente y Recursos Naturales, México, D.F.

Chazdon R.L., Colwell R.K., Denslow J.S. y Guariguata M.R. 1998. Statistical methods for estimating species richness of woody regeneration in primary and secondary rain forests of NE Costa Rica. En: Dallmeier F. y Comiskey J.A. Eds. Forest Biodiversity Research, Monitoring and Modeling: Conceptual Background and Old World Case Studies, pp. 285-309, Parthenon Publishing, Paris.

Crum H.A. 1951. The Appalachian-Ozarkian element in the moss flora of Mexico with a check-list of all known Mexican mosses. 
Tesis de doctorado. University of Michigan, Ann Arbor. 504 pp.

Delgadillo-M C. 1979. Mosses and phytogeography of the Liquidambar forest of Mexico. The Bryologist 82:432-449.

Delgadillo-M C., Bello B. y Cárdenas-S A. 1995. LATMOSS, a catalogue of Neotropical mosses. Monographs in Systematic Botany from the Missouri Botanical Garden 56:1-191. (Disponible en linea: <www.ibiologia.unam.mx/briologia/www/ index/latmoss.html >).

Frego K.A. 2007. Bryophytes as potential indicators of forest integrity. Forest Ecology and Management 242:65-75.

Fritz K.M., Glime J.M., Hribljan J. y Greenwood J.L. 2009. Can bryophytes be used to characterize hydrologic permanence in forested headwater streams? Ecological Indicators 9:681-692.

Glime J.M. y Saxena D. 1991. Uses of Bryophytes. Today \& Tomorrow's Printers \& Publishers, Nueva Delhi.

Mishler B.D. 2003. The biology of bryophytes, with special reference to water. Fremontia 31:34-38.

Nakamura M. y Soberón J. 2008. Use of approximate inference in an index of completeness of biological inventories. Conservation Biology 23:469-474.

Pursell R.A. y Allen B. 2002. Erpodiaceae. Moss flora of Central America. Part 2. Encalyptaceae-Orthotrichaceae. Monographs in Systematic Botany from the Missouri Botanical Garden 90:523531.

Ramírez-Cruz S., Sánchez-González A. y Tejero-Díez D. 2009. La pteridoflora del Parque Nacional Los Mármoles, Hidalgo, México. Boletín de la Sociedad Botánica de México 84:35-44.

Rodríguez P., Soberón J. y Arita H.T. 2003. El componente beta de la diversidad de mamíferos de México. Acta Zoologica Mexica- na 89:241-259.

Rydin H. 2008. Population and community ecology of bryophytes. En: Goffinet B. y Shaw A.J. Eds. Bryophyte Biology, pp. 393444, Cambridge University Press, Nueva York.

Rzedowski J. 1978. Vegetación de México. Editorial Limusa, México, D.F.

Sánchez-González A., Álvarez-Zúñiga E. y Tejero-Díez J.D. 2010. Richness and distribution patterns of ferns and lycopods in Los Mármoles National Park, Hidalgo, Mexico. Journal of the Torrey Botanical Society 137:373-379.

SARH [Secretaría de Agricultura y Recursos Hidráulicos]. 1994. Diagnóstico del Parque Nacional Los Mármoles, estado de Hidalgo. Subsecretaría Forestal y de Fauna Silvestre. Consultores en Ecología y Medio Ambiente. México, D.F.

Sharp A.J., Crum H. y Eckel P.M. 1994. The moss flora of Mexico. Memoirs of the New York Botanical Garden 69:1-1113.

Tuomisto H., Ruokolainen K. y Yli-Halla M. 2003. Dispersal, environment, and floristic variation of western Amazonian forests. Science 299:241-244.

Triantis K.A., Nogués-Bravo D., Hortal J., Borges P.A.V., Adsersen H., Fernández-Palacios J.M., Araújo M.B. y Whittaker R.J. 2008. Measurements of area and the (island) species area relationship: new directions for an old pattern. Oikos 117:15551559.

van Dyke F. 2008. Conservation Biology. Foundations, Concepts, Applications. Springer, Nueva York.

Whittaker R.J., Willis K.J. y Field R. 2001. Scale and species richness: towards a general, hierarchical theory of species diversity. Journal of Biogeography 28:453-470.

Recibido: 25 de abril de 2011

Aceptado: 8 de junio de 2011 